\title{
An Analytic Calculation Method for Delay Time of RC-class Interconnects
}

\author{
Won-Kwang Kal \\ Soongsil Univ. \\ Seoul Korea 156-743 \\ Tel: 082-2-813-0682 \\ Fax: 082-2-822-9872 \\ e-mail: poeun@archi.soongsil.ac.kr
}

\author{
Seok-Yoon Kim \\ Soongsil Univ. \\ Seoul Korea 156-743 \\ Tel: $082-2-820-0682$ \\ Fax: 082-2-822-3622 \\ e-mail: ksy@computing.soongsil.ac.kr
}

\begin{abstract}
This paper presents an analytic $3^{\text {rd }}$ order calculation method, without simulations, for delay time of RC-class circuits, which can be conveniently used to model on-chip interconnects. While the proposed method requires comparable evaluation time to the previous $2^{\text {nd }}$ order calculation method, it ensures more accurate results than those of $2^{\text {nd }}$ order method. The proposed analytic delay calculation method guarantees allowable error tolerance when compared to the results obtained from the AWE technique or HSPICE and has better performance in evaluation time as well as numerical stability. The first algorithm of the proposed method requires 7 moments for the $3^{\text {rd }}$ order approximation and yields accurate delay time approximation. The second algorithm requires 5 moments for the $3^{\text {rd }}$ order approximation and results in shorter evaluation time, the accuracy of which may be less than the first algorithm.
\end{abstract}

\section{INTRODUCTION}

As the device and process technology are improving, delay times of signal propagation due to on chip interconnects become larger than those due to gates. Interconnects are related, directly or indirectly, to the delay time as well as die size, power dissipation in the form of capacitive load and signal coupling due to dense routing. Hence much efforts and researches have been concentrated on the interconnect parameter extraction, modeling and analysis for the accurate estimation and verification of the performance of on chip interconnects. Since interconnects can be analyzed accurately after physical design stage, it is hard to handle interconnects in such a precharacterized form as used for gates. It is a generally accepted approach to use wire load model for interconnect delay based on statistical techniques before physical design and to analyze the interconnect delay using extracted circuit parameters and circuit models after physical design.

In circuit extractors, the modeling of on chip interconnects generally consists of extracting parasitic parameters for a uniform structure, dividing it into small seg- ments, substituting the $T$ - or $\Pi$ - RC lumped model for each segment, and finally cascading each segment. In this method, overall interconnection networks become RC tree, $\mathrm{RC}$ mesh or the compound form of these. The potential problem of this method is that the resultant circuit may be extremely large. Hence, for the delay analysis of interconnects, model order reduction techniques such as Elmore delay[1] and AWE[2] based on the moment matching is largely used rather than the direct analysis method, owing to its lower time complexity.

Elmore delay, the first moment of the impulse response, has been used as a common metric of the RC interconnect signal delay due to its simplicity. As the signal transition time becomes shorter, however, signal bandwidth and maximum operating frequency become higher [3]. Also the current technology trend, such as the device size reduction for highly integrated circuit and the die size increment for high functionality, lengthen the average length of the on chip interconnects, which result in the resistance shielding effect [4]. Owing to the above-mentioned reasons, Elmore delay becomes inadequate delay metric for the high performance circuits.

Contrary to Elmore delay which approximates the interconnect delay using only one time constant, AWE (Asymptotic Waveform Evaluation), a representative method of the order reduction approximates the interconnect delay using $q$ time constants obtained from $2 q$ moments. While AWE requires more evaluation time than Elmore delay, it ensures accurate results comparable to those of the circuit simulator such as SPICE. Generally, for the RC interconnects of the chip whose operating frequency is lower than several $\mathrm{GHz}$, delay times can be calculated within $10 \%$ error tolerance when compared to the results obtained from SPICE [5]. Because of the effectiveness in evaluation time, much efforts were concentrated on the calculation of the delay time of on chip interconnects analytically. Recent works presented an analytic second order approximation algorithm for the on chip interconnect delay time using RC-class [6] or RLC-class [7]. However, considering that second order approximation may result in inaccurate result for high-performance chips and 
systems, it would be agreed that an analytical algorithm for the third order approximation is necessary.

In this paper, we present two analytical algorithms to calculate the delay time of on chip RC networks, based on stable 3 pole approximation. The first proposed algorithm requires 7 moments of the impulse response, and the second one requires 5 moments. Since these algorithms calculate poles without nonlinear iterative method used in AWE, the proposed method improves the evaluation time extremely and overcomes the defect of AWE which is sensitive to numerical noise.

This paper is organized as follows. Following the introduction, in Section 2, we explain Elmore delay and AWE briefly as a background. We pesent two algorithms to calculate poles and residues analytically in Section 3. Since these algorithms calculate poles and residues analytically without iteration, they have several advantages as follows. (1) Evaluation time can be improved extremely. (2) Numerical errors can be reduced. (3) The stability of the resultant circuit model is ensured. The stability of the resultant circuit model is particularly one of the weakness of the model order reduction algorithms such as AWE, which have to use stabilization methods to obtain stable circuit models by means of moment shifting [8] or implementation of passive networks [9]. In Section 4, the results of the proposed method are compared to those of AWE and SPICE. Section 5 concludes this paper.

\section{BACKGROUND}

\section{A. Elmore Delay}

Elmore delay is used as a common delay metric of the on chip RC interconnects due to its simplicity in calculation process. Elmore delay of the on chip RC interconnects having $N$ nodes has $O(N)$ time complexity. For the on chip RC tree, the Elmore delay at some node $i, T_{D i}$, can be expressed as [10]:

$$
T_{D i}=\sum_{k} R_{k i} C_{k}
$$

where $R_{k i}$ is the sum of common resistances of the path between node $k$ to the input node and the path between node $i$ to the input node, and $C_{k}$ is the capacitance at node $k$.

Assuming $h(t)$ to be the unit step response at a certain node, Elmore delay is related to the mean time (or the first moment, $\left.m_{1}\right)$ if we regard the impulse response as a probability density function $\left(\int_{0}^{\infty} h^{\prime}(t) d t=1\right)$, which is expressed as:

$$
T_{D}=m_{1}=\int_{0}^{\infty} t \cdot h(t) d t
$$

It is known that impulse responses of the practical on chip interconnects leans to the left. Hence if we consider the waveform as a probability density function, we can express the following relation:

$$
\text { Mode } \leq \text { Median } \leq \text { Mean }
$$

Since the definition of the delay time is the difference in times between $50 \%$ points of input and output, it is the median rather than mean. However, delay time approximation by Elmore delay uses the mean value of impulse response as the median value and may result in pessimistic estimation, e.g., an upper bound of the real delay time $[10]$.

\section{B. AWE Technique}

The transfer function between a pair of terminal for any linear, lumped, passive circuit is expressed as:

$$
H(s)=\frac{V_{\text {out }}(s)}{V_{\text {in }}(s)}=\frac{1+b_{1} s+b_{2} s^{2}+\ldots+b_{m} s^{m}}{1+a_{1} s+a_{2} s^{2}+\ldots+a_{n} s^{n}}
$$

If we transform Eq.(4) to Eq.(5), we call the coefficient of $i^{\text {th }}$ term as the $i^{\text {th }}$ moment at the node.

$$
H(s)=m_{0}+m_{1} s+m_{2} s^{2}+\ldots
$$

Laplace transform of impulse response in time domain, $h(t)$, is expressed as:

$$
H(s)=\int_{0}^{\infty} h(t) e^{-s t} d t
$$

Hence the expansion of $e^{-s t}$ about $s=0$ by McLaurin Series is expressed as:

$$
\begin{aligned}
H(s) & =\int_{0}^{\infty} h(t)\left[1-s t+\frac{1}{2 !} s^{2} t^{2}-\frac{1}{3 !} s^{3} t^{3}+\ldots\right] d t \\
& =\sum_{k=0}^{\infty} \frac{(-1)^{k}}{k !} s^{k} \int_{0}^{\infty} t^{k} h(t) d t
\end{aligned}
$$

From eq.( 7$), j^{\text {th }}$ coefficient of eq.(5), $m_{j}$, is expressed as:

$$
m_{j}=\frac{(-1)^{j}}{j !} \int_{0}^{\infty} t^{j} h(t) d t
$$

The procedure to approximate poles in AWE consists of performing $2 q$ dc analyses to obtaining $2 q$ moments, and building $q^{t h}$ transfer function using $2 q$ moments. For example, when approximating a complex RC tree circuit to a $3^{\text {rd }}$ order transfer function, 6 moments are required, which can be obtained through 6 successive dc analyses. Using the moment values, eq.(9) is formed, and the 3 roots (e.g. 3 poles) of the characteristic equation, eq.(10), are computed.

$$
\left[\begin{array}{lll}
m_{0} & m_{1} & m_{2} \\
m_{1} & m_{2} & m_{3} \\
m_{2} & m_{3} & m_{4}
\end{array}\right]\left[\begin{array}{l}
a_{3} \\
a_{2} \\
a_{1}
\end{array}\right]=\left[\begin{array}{l}
m_{3} \\
m_{4} \\
m_{5}
\end{array}\right]
$$




$$
a_{3} x^{3}+a_{2} x^{2}+a_{1} x+1=0
$$

LU decomposition is a common method to obtain the vector ' $a$ ' values in eq.(9) and Newton - Raphson or Laguerre method is used to obtain the roots of the characteristic equation [11]. Once the reduction of the circuit to a $q$ pole system has been done, $j^{\text {th }}$ moment can be expressed in terms of poles and residues as:

$$
m_{j}=\sum_{i=1}^{N} \frac{k_{i}}{\left(p_{i}\right)^{j+1}}
$$

where $k_{i}$ is the $i^{\text {th }}$ residue, $p_{i}$ is the $i^{\text {th }}$ pole of the corresponding node. Hence we can calculate the residues using eq. (11).

\section{Analytic Algorithms for $3^{\text {rd }}$ Order APPROXIMATION}

\section{A. Algorithms for 3 Pole Approximation}

Moment matching used in AWE has two main flaws. The first is that the method is very sensitive to the numerical noise produced by computer which has finite precision, due to the divergence characteristic of circuit moments. The second is that it requires non-negligible evaluation time for calculating poles due to embedded iterative procedure such as Newton-Raphson. We discuss numerical noise briefly below, the details of which are dealt in [8]. When reducing big $\mathrm{RC}$ circuits, which have many poles, to simple RC circuits, the effect of high frequency poles to be removed from the circuit may transform the resultant circuits unstable as it gets combined with numerical noise such as truncation error. To overcome this problem, numerous methods have been presented [9], but most of them have a problem in that they require large evaluation times.

The analytical algorithms presented in this section not only overcome the problem discussed above, but also ensure faster evaluation than AWE for obtaining poles. We present two analytical algorithms. The first one (denoted as 'Method -1 ') generates $q^{\text {th }}$ transfer function using $2^{q}$ moments, and ensures accurate delay time approximations. The second one (denoted as 'Method -2') requires $2 q$ moments for approximating $q^{t h}$ order transfer function, and ensures faster evaluation than Method - 1, but its accuracy may be less than that of Method -1 .

A.1 Method-1: Approximation of 3 poles using 7 moments

\section{A.1.1 First pole approximation}

As the order increases, the ratio of successive moments converges to the dominant pole, which is expressed as [12]:

$$
p_{1}=\lim _{i \rightarrow>\infty} \frac{m_{i}}{m_{i+1}} \quad(i: 0,1,2, \ldots)
$$

Eq.(12) can be expressed as Eq.(13) in terms of Eq.(11).

$$
\frac{m_{i}}{m_{i+1}}=p_{1}\left[1+\frac{k_{2}}{k_{1}}\left(\frac{p_{1}}{p_{2}}\right)^{i+1}\left(1-\frac{p_{1}}{p_{2}}\right)\right.
$$

$$
\begin{array}{r}
\left.+\frac{k_{3}}{k_{1}}\left(\frac{p_{1}}{p_{3}}\right)^{i+1}\left(1-\frac{p_{1}}{p_{3}}\right)+\ldots\right] \\
(i: 0,1,2, \ldots)
\end{array}
$$

Since the definition of poles tells $\left|p_{1}\right|<\left|p_{2}\right|<\ldots<$ $\left|p_{n}\right|$, as $i$ increases, Eq. (13) converges to $p_{1}$. Hence the equation for the first pole approximation is expressed as:

$$
p_{1}=\frac{m_{6}}{m_{7}}
$$

\section{A.1.2 Second pole approximation}

For the second pole approximation, we define $A_{i}$ as:

$$
A_{i}=\frac{m_{i}}{m_{i+1}}-\frac{m_{i+1}}{m_{i+2}}
$$

From eq.(13), we obtain eq.(16),

$A_{i}=p_{1}\left[\frac{k_{2}}{k_{1}}\left(\frac{p_{1}}{p_{2}}\right)^{i+1}\left(1-\frac{p_{1}}{p_{2}}\right)^{2}+\frac{k_{3}}{k_{1}}\left(\frac{p_{1}}{p_{3}}\right)^{i+1}\left(1-\frac{p_{1}}{p_{3}}\right)^{2}+\ldots\right]$

and we can derive eq.(17) in terms of eq.(16).

$$
\left|\frac{A_{i}}{A_{i+1}}\right|=\frac{p_{2}}{p_{1}}\left[1+\frac{k_{3}}{k_{2}}\left(\frac{p_{2}}{p_{3}}\right)^{i+1}\left(1-\frac{p_{2}}{p_{3}}\right) \frac{\left(1-\frac{p_{1}}{p_{2}}\right)^{2}}{\left(1-\frac{p_{1}}{p_{3}}\right)^{2}}+\ldots\right]
$$

As $i$ increases, eq.(17) converges to $\frac{p 2}{p 1}$. Hence the equation for the second pole approximation is expressed as:

$$
p_{2}=p_{1} \cdot\left|\frac{A_{4}}{A_{5}}\right|
$$

\section{A.1.3 Third pole approximation}

For the third pole approximation, we define $B_{i}$ as:

$$
B_{i}=\frac{A_{i}}{A_{i+1}}-\frac{A_{i+2}}{A_{i+3}}
$$

From eq.(17), we obtain eq.(20),

$$
\begin{array}{r}
B_{i}=\left(\frac{p_{2}}{p_{1}}\right)\left[\left(\frac{k_{3}}{k_{2}}\right)\left(\frac{p_{2}}{p_{3}}\right)^{i+1}\left(1-\left(\frac{p_{2}}{p_{3}}\right)^{2}\right)\left(1-\frac{p_{2}}{p_{3}}\right)^{2} \frac{\left(1-\frac{p_{1}}{p_{3}}\right)^{2}}{\left(1-\frac{p_{1}}{p_{2}}\right)^{2}}\right. \\
\left.+\left(\frac{k_{4}}{k_{2}}\right)\left(\frac{p_{2}}{p_{4}}\right)^{i+1}\left(1-\left(\frac{p_{2}}{p_{4}}\right)^{2}\right)\left(1-\frac{p_{2}}{p_{4}}\right)^{2} \frac{\left(1-\frac{p_{1}}{p_{4}}\right)^{2}}{\left(1-\frac{p_{1}}{p_{2}}\right)^{2}}+\ldots\right](20)
\end{array}
$$

and we can derive eq.(21) in terms of eq.(20).

$$
\begin{aligned}
& \left|\frac{B_{i}}{B_{i+2}}\right|=\left(\frac{p_{3}}{p_{2}}\right)^{2} \\
& {\left[\frac{1+\left(\frac{k_{4}}{k_{3}}\right)\left(\frac{p_{3}}{p_{4}}\right)^{i+1} \frac{\left(1-\left(\frac{p_{2}}{p_{4}}\right)^{2}\right)\left(1-\frac{p_{2}}{p_{4}}\right)^{2}\left(1-\frac{p_{1}}{p_{4}}\right)^{2}}{\left(1-\left(\frac{p_{2}}{p_{3}}\right)^{2}\right)\left(1-\frac{p_{2}}{p_{3}}\right)^{2}\left(1-\frac{p_{1}}{p_{3}}\right)^{2}}+\ldots}{1+\left(\frac{k_{4}}{k_{3}}\right)\left(\frac{p_{3}}{p_{4}}\right)^{i+3} \frac{\left(1-\left(\frac{p_{2}}{p_{4}}\right)^{2}\right)\left(1-\frac{p_{2}}{p_{4}}\right)^{2}\left(1-\frac{p_{1}}{p_{4}}\right)^{2}}{\left(1-\left(\frac{p_{2}}{p_{3}}\right)^{2}\right)\left(1-\frac{p_{2}}{p_{3}}\right)^{2}\left(1-\frac{p_{1}}{p_{3}}\right)^{2}}+\ldots}\right]}
\end{aligned}
$$

As $i$ increases, eq. (21) converges to $\left(\frac{p 3}{p^{2}}\right)^{2}$. Hence the equation for the third pole approximation is expressed as:

$$
p_{3}=p_{2} \sqrt{\frac{B_{0}}{B_{2}}}
$$




\section{A.2 Method-2: Approximation of 3 poles using 5 moments}

\section{A.2.1 First pole approximation}

The equation for approximating the first pole using 5 moments is expressed in terms of eq.(13) as:

$$
p_{1}=\frac{m_{4}}{m_{5}}
$$

\section{A.2.2 Second pole approximation}

The equation for approximating the second pole using 5 moments is expressed in terms of eq.(17) as:

$$
p_{2}=p_{1} \cdot\left|\frac{A_{2}}{A_{3}}\right|
$$

\section{A.2.3 Third pole approximation}

For the third pole approximation using 5 moments, we define $C_{i}$ as:

$$
C_{i}=\frac{A_{i}}{A_{i+1}}-\frac{A_{i+1}}{A_{i+2}}
$$

From eq.(17), we obtain eq.(26),

$$
\begin{aligned}
C_{i} & =\frac{p_{2}}{p_{1}}\left[\left(\frac{k_{3}}{k_{2}}\right)\left(\frac{p_{2}}{p_{3}}\right)^{i+1}\left(1-\frac{p_{2}}{p_{3}}\right)^{2} \frac{\left(1-\frac{p_{1}}{p_{3}}\right)^{2}}{\left(1-\frac{p_{1}}{p_{2}}\right)^{2}}\right. \\
& \left.+\left(\frac{k_{4}}{k_{2}}\right)\left(\frac{p_{2}}{p_{4}}\right)^{i+1}\left(1-\frac{p_{2}}{p_{4}}\right)^{2} \frac{\left(1-\frac{p_{1}}{p_{4}}\right)^{2}}{\left(1-\frac{p_{1}}{p_{2}}\right)^{2}}+\ldots\right]
\end{aligned}
$$

and we can derive eq.(27) in terms of eq.(26).

$$
\left|\frac{C_{i}}{C_{i+1}}\right|=\left(\frac{p_{3}}{p_{2}}\right)\left[\frac{1+\left(\frac{k_{4}}{k_{3}}\right)\left(\frac{p_{3}}{p_{4}}\right)^{i+1} \frac{\left(1-\frac{p_{2}}{p_{4}}\right)^{2}\left(1-\frac{p_{1}}{p_{4}}\right)}{\left(1-\frac{p_{2}}{p_{3}}\right)^{2}\left(1-\frac{p_{1}}{p_{3}}\right)}+\ldots}{1+\left(\frac{k_{4}}{k_{3}}\right)\left(\frac{p_{3}}{p_{4}}\right)^{i+2} \frac{\left(1-\frac{p_{2}}{p_{4}}\right)^{2}\left(1-\frac{p_{1}}{p_{4}}\right)}{\left(1-\frac{p_{2}}{p_{3}}\right)^{2}\left(1-\frac{p_{1}}{p_{3}}\right)}+\ldots}\right]
$$

As $i$ increases, eq. (27) converges to $\frac{p 3}{p 2}$. Hence the equation for the third pole approximation using 5 moments is expressed as:

$$
p_{3}=p_{2} \cdot\left|\frac{C_{0}}{C_{1}}\right|
$$

\section{B. Stability of the Proposed Method}

For linear, finite and lumped circuits, the stability (in the sense of Lyapunov) can be analyzed by searching the poles of circuits [13,14]. For the circuit response to converge at $t=\infty$, all the poles must be on the left hand side of the Laplace plain, which implies that every pole has a negative real part. We show that all the poles obtained by the proposed algorithms, Method-1 and Method-2, have negative real parts intrinsically in the following:

Proposition 1: Poles obtained by Method - 1 and Method -2 are real numbers.

This proposition follows from the fact that the proposed algorithms obtain the poles through the multiplication and division of real numbers.

Proposition2: Poles obtained by Method -1 and Method -2 are negative numbers.
Since the moments of RC-circuit always satisfy $m_{2 i}>$ $0, m_{2 i+1}<0(i: 0,1,2, \ldots)$, the ratio of successive moments is a negative number. Hence, $p_{1} \mathrm{~s}$ in eq.(14) and eq.(23) are negative numbers, and so are $p_{2} \mathrm{~s}$ in eq.(18) and eq.(24), and $p_{3}$ s in eq.(22) and eq.(28).

\section{Algorithm for Residue Approximation}

For a system with $N$ poles, we can obtain $N$ residues from the following equation [2]:

$$
\left[\begin{array}{cccc}
1 & 1 & \cdots & 1 \\
\frac{1}{p_{1}} & \frac{1}{p_{2}} & \cdots & \frac{1}{p_{N}} \\
\vdots & \vdots & \ddots & \vdots \\
\frac{1}{p_{1}^{N-1}} & \frac{1}{p_{2}^{N-1}} & \cdots & \frac{1}{p_{N}^{N-1}}
\end{array}\right]\left[\begin{array}{c}
k_{1} \\
k_{2} \\
\vdots \\
k_{N}
\end{array}\right]=\left[\begin{array}{c}
m_{0} \\
m_{1} \\
\vdots \\
m_{N-1}
\end{array}\right]
$$

For simplicity, we transform eq.(29) to:

$$
\begin{aligned}
& k_{1}=\frac{-m_{2}+\left(D_{2}+D_{3}\right) \cdot m_{1}-D_{2} D_{3} \cdot m_{0}}{D_{1}\left(D_{3}-D_{1}\right)\left(D_{1}-D_{2}\right)} \\
& k_{2}=\frac{-m_{2}+\left(D_{3}+D_{1}\right) \cdot m_{1}-D_{3} D_{1} \cdot m_{0}}{D_{2}\left(D_{1}-D_{2}\right)\left(D_{2}-D_{3}\right)} \\
& k_{3}=\frac{-m_{2}+\left(D_{1}+D_{2}\right) \cdot m_{1}-D_{1} D_{2} \cdot m_{0}}{D_{3}\left(D_{2}-D_{3}\right)\left(D_{3}-D_{1}\right)}
\end{aligned}
$$

where $D_{i}=\frac{1}{p_{i}}$. Hence we can calculate residues algebraically using eq.(30).

\section{Delay Time Estimation}

In terms of the poles and residues, the output waveform at a circuit node can be approximated as:

$$
h(t)=k_{1} e^{p_{1} t}+k_{2} e^{p_{2} t}+k_{3} e^{p_{3} t}
$$

By the definition of the delay time, which is the time between $50 \%$ points of input and output waveforms, the delay time of the step input, $t_{D}$, is calculated from the following equation:

$$
k_{1} e^{p_{1} t_{D}}+k_{2} e^{p_{2} t_{D}}+k_{3} e^{p_{3} t_{D}}=0.5 V_{\text {out }}
$$

\section{Experimental Results}

In this section, we present the experimental results to verify the accuracy and efficiency of the proposed method. We simulated practical $\mathrm{RC}$ tree circuits having different number of nodes, from 100 to 1000 . The simulation was performed on SUN UltraSparc $-V$ workstation. The contents of following table- 1 are the delay times for each circuit at the fan-out nodes. From the table-1, we know that the proposed analytic algorithms guarantee allowable error tolerance when compared to the results obtained from AWE and HSPICE.

Evaluation time comparison is shown in table- 2 and table-3. As shown in table- 2 and table- 3 , the proposed 
TABLE I

Delay times at FAN-OUt NODES For a RAMP inPut(INPUt Rise time: 1Ns) (Relative error(\%) COMPared to the HSPICE are indicated in Parantheses.)

\begin{tabular}{|c|c|c|c|c|}
\hline \# of nodes & HSPICE & AWE & Method-1 & Method-2 \\
\hline 100 & $1.2128 \mathrm{e}-11$ & $1.2333 \mathrm{e}-11(1.69)$ & $1.2333 \mathrm{e}-11(1.69)$ & $1.2333 \mathrm{e}-11(1.69)$ \\
\hline 200 & $5.0442 \mathrm{e}-11$ & $5.0567 \mathrm{e}-11(0.25)$ & $5.0568 \mathrm{e}-11(0.25)$ & $5.0567 \mathrm{e}-11(0.25)$ \\
\hline 300 & $1.0634 \mathrm{e}-10$ & $1.0634 \mathrm{e}-10(0)$ & $1.0634 \mathrm{e}-10(0)$ & $1.0634 \mathrm{e}-10(0)$ \\
\hline 400 & $1.8282 \mathrm{e}-10$ & $1.8171 \mathrm{e}-10(-0.61)$ & $1.8171 \mathrm{e}-10(-0.61)$ & $1.8172 \mathrm{e}-10(-0.60)$ \\
\hline 500 & $2.7434 \mathrm{e}-10$ & $2.7373 \mathrm{e}-10(-0.22)$ & $2.7374 \mathrm{e}-10(-0.22)$ & $2.7375 \mathrm{e}-10(-0.22)$ \\
\hline 600 & $3.7438 \mathrm{e}-10$ & $3.7502 \mathrm{e}-10(0.17)$ & $3.7504 \mathrm{e}-10(0.18)$ & $3.7506 \mathrm{e}-10(0.18)$ \\
\hline 700 & $4.9196 \mathrm{e}-10$ & $4.8804 \mathrm{e}-10(-0.8)$ & $4.8807 \mathrm{e}-10(-0.79)$ & $4.8812 \mathrm{e}-10(-0.78)$ \\
\hline 800 & $6.2669 \mathrm{e}-10$ & $6.2076 \mathrm{e}-10(-0.95)$ & $6.2159 \mathrm{e}-10(-0.81)$ & $6.2253 \mathrm{e}-10(-0.66)$ \\
\hline 900 & $7.7612 \mathrm{e}-10$ & $7.6538 \mathrm{e}-10(-1.38)$ & $7.5458 \mathrm{e}-10(-2.78)$ & $7.5512 \mathrm{e}-10(-2.71)$ \\
\hline 1000 & $9.2790 \mathrm{e}-10$ & $9.0804 \mathrm{e}-10(-2.14)$ & $9.0793 \mathrm{e}-10(-2.15)$ & $9.0822 \mathrm{e}-10(-2.12)$ \\
\hline
\end{tabular}

TABLE II

Calculation times of 3 poles(Averaged over 10 EXPERIMENTS, UNIT:MS)

(Speed Ratios compared to the existing AWE method are INDICATED IN PARENTHESES)

\begin{tabular}{|c|c|c|}
\hline AWE & Method-1 & Method-2 \\
\hline $1.8551(1)$ & $0.0221(88)$ & $0.008(232)$ \\
\hline
\end{tabular}

TABLE III

Calculation times of 3 Residues (Averaged over 10 EXPERIMENTS, UNIT:MS)

(Speed ratios compared to the existing AWE method are INDICATED IN PARENTHESES)

\begin{tabular}{|c|c|c|}
\hline AWE & Method-1 & Method-2 \\
\hline $0.2702(1)$ & $0.0063(43)$ & $0.0086(31)$ \\
\hline
\end{tabular}

methods are faster than AWE, the speed-up of which is of 2-3 orders for evaluating the poles and of 2 orders for evaluating the residues. The reason that $M$ ethod -1 requires longer evaluation time for pole computation than Method-2 is that Method - 1 handles more number of moments than Method-2. In case of computing residues, both proposed methods yield about the same speed-up compared to the AWE results.

\section{CONCLUSION}

In this paper, we presented an analytic algorithm to compute the poles and residues of on-chip RC circuits, which not only overcomes numerical sensitivity and instability problems of moment matching methods, but requires less evaluation time than AWE. Although the proposed method is restricted to the $3^{\text {rd }}$ order approximation, which yields good results for most practical RC circuits, it is easy to expand the equations to higher order ones due to its regularity when technology compels.

As the order of equation increases, the proposed method will guarantee allowable error tolerances compared to the results obtained from AWE and the evaluation time will shrink superlinearly compared to AWE. Hence the proposed method is expected to improve the performance in electrical and physical design such as placement \& routing which requires iterations of delay time calculation.

\section{REFERENCES}

[1] W. C. Elmore, "The Transient Analysis of Damped Linear Networks with Particular Regard to Wideband Amplifiers," J. Applied Physics, vol. 19(1), 1948.

[2] L. T. Pillage and R. A. Rohrer, "Asymptotic Waveform Evaluation for Timing Analysis," IEEE Trans, on Computer Aided Design, vol. 9, no. 4, April 1990.

[3] N. Gopal, D. P. Neikirk and L. T. Pillage, "Evaluating On-chip RC-Interconnect Using Moment-Matching Approximations," In Proceedings of IEEE/ACM International Conference on Computer Aided Design, November 1991.

[4] Jessica Qian, Satyamurthy Pullela and Lawrence Pillage "Modeling of "Effective Capacitance" for the RC interconnect of CMOS Gates," IEEE Tran. on Computer-Aided-Design of Integrated Circuits and Systems, Vol. 13, No. 12, Dec. 1994.

[5] L. T. Pileggi, "Coping with $\mathrm{RC}(\mathrm{L})$ Interconnect Design Headaches," In Proceedings of IEEE/ACM Int'l Conference on Computer Aided Design, pp. 246-253, Nov. 1995.

[6] B. Tutuianu, F. Dartu and L. T. Pileggi, "An Explicit RCCircuit Delay Approximation Based on the First Three Moments of the Impulse Response," In Proceedings of the $33 \mathrm{th}$ ACM/IEEE Design Automation Conference, 1996.

[7] Andrew B. Kahng and Sudhakar Muddu, "An Analytical Delay Model for RLC Interconnects," Proc. 33th ACM/IEEE Design Automation Conf., pp. 237-240, 1996.

[8] D. F. Anastasakis, N. Gopal, S. Y. Kim and L. T. Pillage, "Enhancing the Stability of Asymptotic Waveform Evaluation for Digital Interconnect Circuit Applications," IEEE Trans, on Computer Aided Design, vol. 13, no. 6, pp. 729-736, June 1994.

[9] A. Odabasioglu, M. Celik and L. T. Pileggi, "PRIMA: Passive Reduced-Order Interconnect Macromodeling Algorithm," IEEE Trans, on Computer Aided Design, vol. 17, no. 8, pp. 645-654, August 1998.

[10] R. Gupta, B. Tutuianu and L. T. Pillage, "The Elmore Delay as a Bound for RC Trees with Generalized Input Signals," IEEE Trans. on Computer Aided Design of Integrated Circuits and Systems, vol. 16, no. 1, January 1997.

[11] A. Ralston and P. Rabinowitz, A First Course in Numerical Analysis, McGraw-Hill Book Co., 1978. 
[12] D. Anastasakis, N. Gopal, S. Y. Kim and L. T. Pillage, "On the Stability of Approximations in Asymptotic Waveform Evaluation," In Proceedings of the 29th ACM/IEEE Design Automation Conference, 1992
[13] S. K. Mitra, Analysis and Synthesis of Linear Active Networks, John Wiley \&S Sons, Inc., 1969.

[14] S. Y. Kim, "A Practical Test for Unconditional Stability of Linear N-port Networks," Korea Information Science Society, vol. 23 no. 7, pp. 701-708, Jul. 1996. 